\title{
Cosmic-ray acceleration efficiency of the supernova remnants RCW 86 and SNR 0509-67.5
}

\author{
Eveline Helder*t \\ Utrecht University \\ E-mail: e.a.heldereastro-uu.nl \\ Jacco Vink \\ Utrecht University \\ E-mail: j.vink@astro-uu.nl
}

\begin{abstract}
We study the effect of cosmic-ray acceleration on the plasma temperature behind shock fronts, in order to characterize the cosmic-ray acceleration efficiencies. We combine post-shock plasma temperatures with shock velocities and show that this leads to a direct measurement of the cosmicray pressure behind the shock front. Additionally, we tie this pressure directly to the fraction of energy taken away from the shock by escaping particles. We show that at least some young supernova remnants transfer a significant amount of their energy into cosmic rays and these cosmic rays take away a substantial amount of the supernova energy by escaping the remnant.
\end{abstract}

25th Texas Symposium on Relativistic Astrophysics - TEXAS 2010

December 06-10, 2010

Heidelberg, Germany

\footnotetext{
* Speaker.

Corresponding author
} 


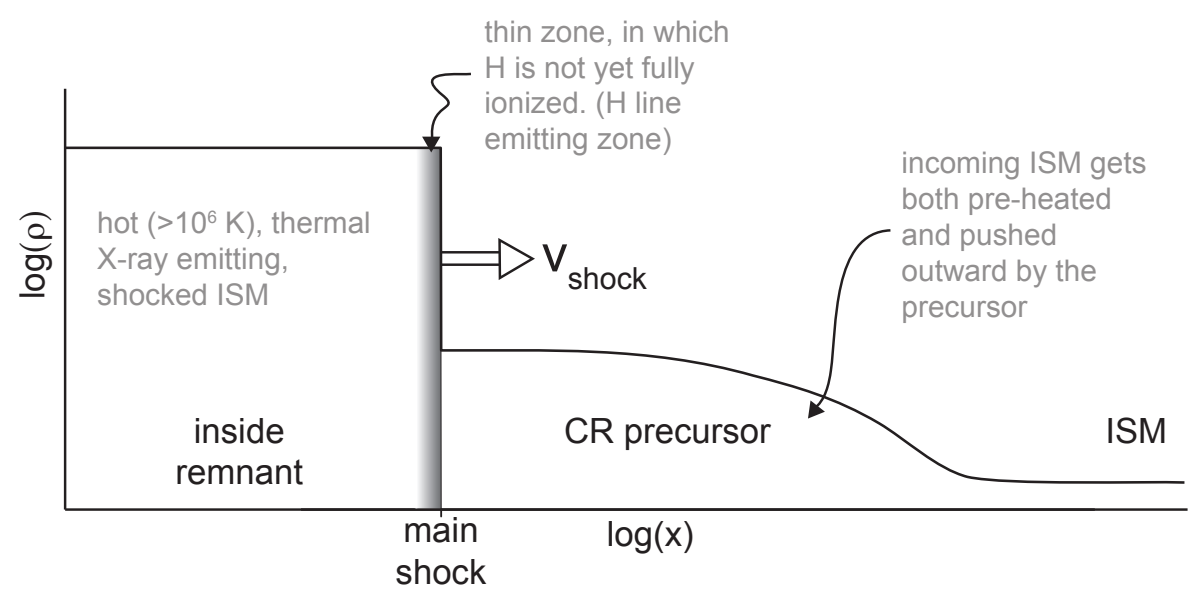

Figure 1: Structure of a Balmer-dominated cosmic-ray modified shock [taken from 6].

\section{Introduction}

Cosmic rays contribute approximately one third to the Galactic energy density, and escape from the Galaxy in 15.6 \pm 1.6 Myr [22]. To compensate for these losses, Galactic supernovae should transfer a significant amount of their kinetic energy into cosmic rays, assuming they are the main sources for Galactic cosmic rays. The process which is thought to accelerate cosmic rays is called diffusive shock acceleration. This process operates at shock fronts, and causes charged particles to scatter up and down the shock front, gaining energy each time they cross the shock front.

Evidence for particle acceleration at the shock fronts of supernova remnants comes from thin $\mathrm{X}$-ray synchrotron rims at the shock fronts of several supernova remnants, revealing the presence of $\mathrm{TeV}$ electrons [13]. Other evidence comes from the detection of several supernova remnants in $\mathrm{GeV}$ and $\mathrm{TeV} \gamma$-ray energies $[3,1]$.

Once a shock accelerates cosmic rays, the structure of the shock changes: a cosmic-ray precursor will develop, which pushes out and pre-heats the incoming medium (Figure 1). As a result, the Mach number of the subshock (main shock) will decrease and the plasma behind the shock front will be cooler than for a non-accelerating shock with the same shock velocity. The mean plasma temperature $(T)$ for a shock without cosmic rays can be calculated from the shock velocity $\left(v_{\mathrm{s}}\right)$ : $k T=\frac{3}{16} \mu m_{\mathrm{p}} v_{\mathrm{s}}^{2}$, in which $\mu$ is the mean particle mass ( 0.6 for fully ionized solar abundances) and $m_{\mathrm{p}}$ is the proton mass.

Hence, we can use the shock velocity, combined with the post-shock temperature, to characterize the cosmic-ray acceleration efficiency. In this proceeding, we describe observations of shocks associated with the supernova remnants RCW 86 and SNR 0509-67.5. These were carried out to measure the cosmic-ray acceleration efficiency. We also compare these results with what has been found for the old Cygnus Loop supernova remnant, and the 1E 0102-72.3 supernova remnant in the Small Magellanic Cloud. Furthermore, we show how we can use those observations to calculate how much energy the shock converts into cosmic-ray energy. Moreover, we use the observations to calculate how much of this cosmic-ray energy is released into the ambient medium. 


\section{Observations}

For most remnants, the electron temperature of the plasma is relatively easily determined from their thermal X-ray spectra. However, as the electron and proton temperature can be rather different [5] and in most extreme cases can be proportional to the particle mass [15], it is non-trivial how the electron temperature relates to the mean plasma temperature behind the shock front. Also, the X-ray spectrum is often summed over some distance behind the shock front, as 1) the angular resolution of X-ray telescopes is of the order of $\geq 0.5$ ", and 2) we need to collect enough photons to get a spectrum for which we can determine the electron temperature reliably. This means that the measured electron temperature has already undergone some equilibration at the location where we measure it, and one needs to correct for that.

For some remnants, the proton temperature can be estimated from their optical spectra. These are so-called Balmer dominated remnants, and the hydrogen lines in their spectrum consist of two superimposed components [for a review, see 10]. The narrow component is emitted after excitation of the hydrogen atoms within the shock and its width is representative of the temperature of the ambient medium [however, see 16]. The broad component is emitted after charge exchange and therefore its width provides an estimate for the post-shock proton temperature. As the neutral hydrogen is ionized very quickly after entering the shock front, the hydrogen line emission is strongly tied to the physical conditions right behind the shock front. Also, the proton temperature is close to the mean plasma temperature, independent of the measure of temperature equilibration behind the shock, as the mean particle mass is close to the proton mass.

The shock velocity can be measured through proper motion studies of the shocks. However, this method relies on a known distance towards the supernova remnant, which is often hard to determine.

\subsection{RCW 86}

For RCW 86, we used VLT/FORS2 observations to obtain an optical spectrum [9] from the shock front in the northeast, that is known to emit X-ray synchrotron emission [20]. From the broad component, we determine a post-shock proton temperature of $2.3 \pm 0.3 \mathrm{keV}$. In addition, we measured the proper motion of the shock front to be $0.5 \pm 0.2 \% / y r$, using two epochs of Chandra observations. Together with a distance of $2.5 \pm 0.5 \mathrm{kpc}$, this leads to a velocity of $6000 \pm 2800 \mathrm{~km} / \mathrm{s}$. We caution that the shock velocity that is physically related to the obtained proton temperature is more reliably measured from an optical proper motion study. Using the Chandra proper motion measurement, we find that $\beta \equiv \frac{16 k T_{\mathrm{p}}}{3 \mu m_{\mathrm{p}} v_{\mathrm{s}}^{2}}<0.31$. Note that $\beta=1$ for a shock in thermal equilibrium without cosmic-ray acceleration.

\subsection{SNR 0509-67.5}

We obtained VLT/FORS2 observations for the LMC remnant 0509-67.5 as well [8], resulting in $k T_{\mathrm{p}}$ of $15.9 \pm 0.9 \mathrm{keV}$ for the SW shock, for which we obtained a lower limit on the shock velocity of $5000 \mathrm{~km} / \mathrm{s}$, leading to $\beta \leq 0.58$. This velocity is based on the line broadening as obtained with the XMM-Newton/RGS instrument [12], together with the age as determined from light echos [17] and models for the evolution of a supernova remnant. A preliminary proper motion study with two epochs of Chandra observations resulted even in a somewhat higher shock velocity. 


\subsection{Cygnus Loop}

For the old ( $\sim 10,000$ year) Cygnus Loop remnant, a similar study has been carried out [18], using the electron temperature. The shocks of this remnant have significantly slowed down $\left(v_{\mathrm{s}}<300\right.$ $\mathrm{km} / \mathrm{s}$ ) and since there seems to be a general trend that shocks with $v_{\mathrm{s}}<500 \mathrm{~km} / \mathrm{s}$ are equilibrated [5] one can use electron temperatures in order to characterize the plasma temperature. The electron temperatures indicate that there is negligible cosmic-ray pressure in the Cygnus Loop remnant.

\subsection{SNR 1E 0102-72.3}

SNR 0102-72.3 is located in the Small Magellanic Cloud, at a distance of $60 \mathrm{kpc}$. Combining Einstein, ROSAT and Chandra data in a proper motion study resulted in a shock velocity of 6000 $\mathrm{km} / \mathrm{s}$ [11]. The electron temperature close to the shock front is, however, less than $1 \mathrm{keV}$. Taking temperature equilibration processes and adiabatic decompression into account, one would expect an electron temperature of at least $2.5 \mathrm{keV}$ for this shock velocity [11]. Therefore, the actual temperature divided by the expected temperature is $\leq 0.4$ for this remnant. We take this value as substitute for $\beta$ in the following paragraph.

\section{Interpretation}

To interpret the discrepancies between the expected temperatures expected and the true temperatures, we start with the equations of conservation of mass, momentum and energy. We add cosmic-ray pressure and energy to these equations, in which the energy is the cosmic-ray energy flux escaping the shock and the cosmic-ray pressure is the fraction of the pressure behind the shock front, contributed by cosmic rays. Moreover, we assume the shock to be in steady state and to be plane parallel. We note that this approach is a first step in interpreting our measurements in terms of a quantitative estimation of the cosmic-ray acceleration efficiency.

We evaluate the shock conditions at 3 locations: ' 0 ' is far ahead of the shock (outside of the remnant), ' 1 ' is right in front of the main (gas) shock and ' 2 ' is just behind the main shock. The gas is adiabatically compressed and heated in the precursor, after which it gets shock heated at the main shock. The cosmic-ray pressure, however, is constant over the main shock, and negligible far ahead of the shock. Working out the equations [21], one sees that the comic-ray pressure is one-to-one related to the energy flux escaping the shock. The only other parameter that determines this relation is the equation-of-state of the cosmic-rays. This equation of state is a function of the shape of the cosmic-ray spectrum, as a harder spectrum results in a equation-of-state closer to $4 / 3$ (for a relativistic plasma), and a softer spectrum results in an equation-of-state closer to $5 / 3$ (a non-relativistic plasma). Figure 2 shows the relation for escaping energy flux and cosmic-ray pressure for an equation-of-state of the cosmic rays of $4 / 3$. The value for those quantities can be determined by $\beta$. Overdrawn are the values for $\beta$ for the remnants described in these proceedings. Note that the shocks do not only produce a significant amount of cosmic-ray pressure, they also lose a substantial amount of their energy by cosmic rays that escape from the shock. This may be a possible reason why a young supernova remnant like Cassiopeia A does not seem to have a high cosmic-ray energy [1]. 


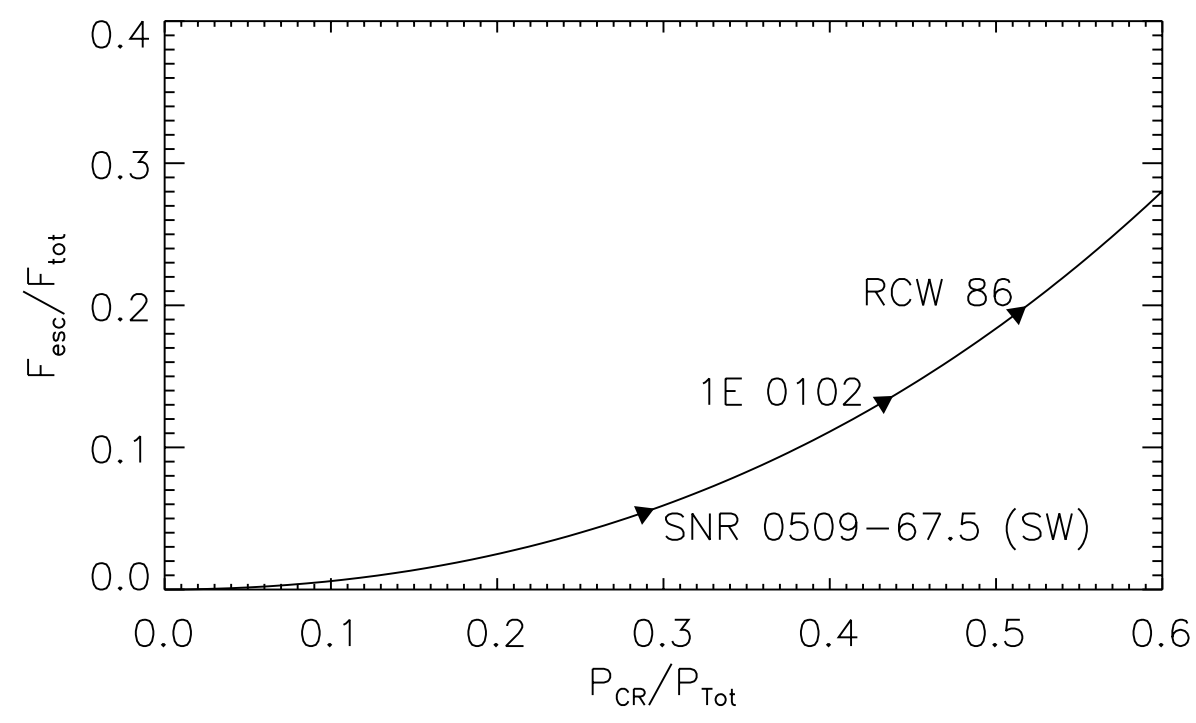

Figure 2: Cosmic-ray pressure and energy flux on the $x$ and $y$ axes respectively. The relation is plotted for a shock with a Mach number of 400 and an equation of state for the cosmic rays of $4 / 3$. The $\beta$-values for the remnants mentioned in these proceedings are on the curve, and indicated with an upwards arrow, as they are conservative estimates.

\section{Temperature equilibration}

In calculating the lower limits for $\beta$ for RCW 86 and SNR 0509-67.5, we assumed the electron and proton temperature to be in equilibrium. This is a conservative assumption, as we assume a significant fraction of the post-shock pressure to be contained in the electrons which could actually be cosmic-ray pressure if we knew the electron temperature to be low. Recent studies, showed the electron temperature to be only a fraction of the proton temperature behind high Mach number shocks [5, 19]. These studies, however, do not take into account the effects of cosmic-ray acceleration. This is probably a good assumption for the shocks in their study, as they are generally bright in $\mathrm{H} \alpha$, implying a large neutral density outside the shock and neutrals are thought to hamper cosmic-ray acceleration by damping the turbulence needed to scatter the particles. As RCW 86 and SNR 0509-67.5 have faint $\mathrm{H} \alpha$ emission, and are active accelerators, it is unclear whether the electron and proton temperature are as far out of equilibrium as predicted [5, 19]. There are reasons to believe that efficient cosmic-ray acceleration changes the measure of electron-proton temperature equilibration. First, the cosmic-ray precursor heats the ambient medium before the medium reaches the main shock. This lowers the Mach number of the main shock, and therewith possibly altering the measure of electron to proton temperature equilibration [4]. Secondly, as the total compression ratio over the shock increases, the post-shock density increases, leading to faster equilibration though Coulomb equilibration [14].

Recently, we studied the electron to proton temperature equilibration for a number of shocks in RCW 86 [7]. RCW 86 is an excellent source for this study, as it has a large variety in shock velocities, and has shown to be a cosmic-ray accelerator [2]. We based the proton temperatures on published widths of the broad component of the $\mathrm{H} \alpha$ lines, taken from different shocks of the 
remnant. The electron temperature was measured from XMM-Newton spectra, and corrected for equilibration effects that occurred between the shock and the location where the spectrum was extracted. This study showed does not show a very clear trend of the measure of temperature equilibration as function of $v_{\mathrm{s}}$. However, more study is necessary to conclude that this is caused by cosmic-ray acceleration processes.

\section{Conclusions}

We described several studies that have characterized the cosmic-ray acceleration efficiency of supernova remnants by comparing the shock velocities of the remnants with post-shock temperatures. Moreover, we describe studies about the interpretation of these observations. Based on these studies, we can reach the following conclusions:

- For three young remnants, a significant cosmic-ray acceleration efficiency has been measured by comparing their shock speeds with their post-shock temperatures.

- These remnants also lose a substantial fraction of their cosmic rays to the ambient medium, contributing to the cosmic-ray spectrum as observed on earth.

- The old Cygnus Loop remnant has negligible cosmic-ray acceleration.

- Knowing the electron to proton temperature ratio behind cosmic-ray accelerating shock fronts is important to further constrain the cosmic-ray acceleration efficiency.

- A preliminary study shows that cosmic-ray acceleration might affect the measure of temperature equilibration behind shock fronts.

\section{References}

[1] A. A. Abdo et al. ApJ, 710:L92-L97, February 2010.

[2] F. Aharonian et al. ApJ, 692:1500-1505, February 2009.

[3] F. A. Aharonian et al. Nature, 432:75-77, November 2004.

[4] A. M. Bykov and Yu. A Uvarov. JETP, 88:465-475, 1999.

[5] P. Ghavamian, J. M. Laming, and C. E. Rakowski. ApJ, 654:L69-L72, January 2007.

[6] E. A. Helder. PhD thesis, Utrecht University, September 2010.

[7] E. A. Helder et al. ApJ submitted. 2011.

[8] E. A. Helder, D. Kosenko, and J. Vink. ApJ, 719:L140-L144, August 2010.

[9] E. A. Helder, J. Vink, C. G. Bassa, A. Bamba, J. A. M. Bleeker, S. Funk, P. Ghavamian, K. J. van der Heyden, F. Verbunt, and R. Yamazaki. Science, 325:719-, August 2009.

[10] K. Heng. PASA, 27:23-44, March 2010. 
[11] J. P. Hughes, C. E. Rakowski, and A. Decourchelle. ApJ, 543:L61-L65, November 2000.

[12] D. Kosenko, J. Vink, S. Blinnikov, and A. Rasmussen. A\&A, 490:223-230, October 2008.

[13] K. Koyama, R. Petre, E. V. Gotthelf, U. Hwang, M. Matsuura, M. Ozaki, and S. S. Holt. Nature, 378:255-+, November 1995.

[14] D. J. Patnaude, D. C. Ellison, and P. Slane. ApJ, 696:1956-1963, May 2009.

[15] J. C. Raymond, W. P. Blair, and K. S. Long. ApJ, 454:L31+, November 1995.

[16] J. C. Raymond et al. ApJ accepted. 2011.

[17] A. Rest, N. B. Suntzeff, K. Olsen, J. L. Prieto, R. C. Smith, D. L. Welch, A. Becker, M. Bergmann, A. Clocchiatti, K. Cook, A. Garg, M. Huber, G. Miknaitis, D. Minniti, S. Nikolaev, and C. Stubbs. Nature, 438:1132-1134, December 2005.

[18] G. Salvesen, J. C. Raymond, and R. J. Edgar. ApJ, 702:327-339, September 2009.

[19] M. van Adelsberg, K. Heng, R. McCray, and J. C. Raymond. ApJ, 689:1089-1104, December 2008.

[20] J. Vink, J. Bleeker, K. van der Heyden, A. Bykov, A. Bamba, and R. Yamazaki. ApJ, 648:L33-L37, September 2006.

[21] J. Vink, R. Yamazaki, E. A. Helder, and K. M. Schure. ApJ, 722:1727-1734, October 2010.

[22] N. E. Yanasak, M. E. Wiedenbeck, R. A. Mewaldt, A. J. Davis, A. C. Cummings, J. S. George, R. A. Leske, E. C. Stone, E. R. Christian, T. T. von Rosenvinge, W. R. Binns, P. L. Hink, and M. H. Israel. ApJ, 563:768-792, December 2001. 